Keywords: Silicon, solar cell, anti-reflective coating, biomimetic "moth eye"

Retention: Permanent

\title{
Performance of "Moth Eye" Anti-Reflective Coatings for Solar Cell Applications
}

Dr. Elliot A. Clark

Savannah River National Laboratory

Dr. Marie C. Kane

Sandia National Laboratories

Prof. Peng Jiang

University of Florida

February 2011

Savannah River National Laboratory Savannah River Nuclear Solutions, LLC Aiken, SC 29808

Prepared for the U.S. Department of Energy under contract number DE-AC09-08SR22470.

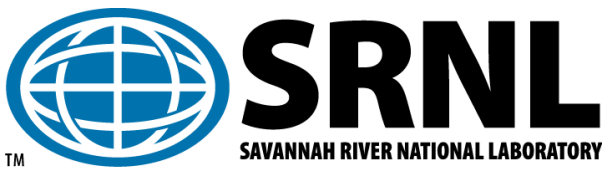




\section{DISCLAIMER}

This work was prepared under an agreement with and funded by the U.S. Government. Neither the U.S. Government or its employees, nor any of its contractors, subcontractors or their employees, makes any express or implied:

1. warranty or assumes any legal liability for the accuracy, completeness, or for the use or results of such use of any information, product, or process disclosed; or

2. representation that such use or results of such use would not infringe privately owned rights; or

3. endorsement or recommendation of any specifically identified commercial product, process, or service.

Any views and opinions of authors expressed in this work do not necessarily state or reflect those of the United States Government, or its contractors, or subcontractors.

Printed in the United States of America

Prepared for

U.S. Department of Energy 


\section{EXECUTIVE SUMMARY}

An inexpensive, effective anti-reflective coating (ARC) has been developed at the University of Florida to significantly enhance the absorption of light by silicon in solar cells. This coating has nano-scale features, and its microstructure mimics that of various night active insects (e.g. a moth's eye). It is a square array of pillars, each about $700 \mathrm{~nm}$ high and having a diameter of about $300 \mathrm{~nm}$. Samples of silicon having this coating were exposed either to various combinations of either elevated temperature and humidity or to gamma irradiation $\left({ }^{60} \mathrm{Co}\right)$ at the Savannah River National Laboratory, or to a broad spectrum ultraviolet light and to a $532 \mathrm{~nm}$ laser light at the University of Florida. The anti-reflective properties of the coatings were unaffected by any of these environmental stresses, and the microstructure of the coating was also unaffected. In fact, the reflectivity of the gamma irradiated ARC became lower (advantageous for solar cell applications) at wavelengths between 400 and $1000 \mathrm{~nm}$. These results show that this coating is robust and should be tested in actual systems exposed to either weather or a space environment. Structural details of the ARCs were studied to optimize their performance. Square arrays performed better than hexagonal arrays- the natural moth-eye coating is indeed a square array. The optimal depth of the templated nanopillars in the ARC was investigated. A wet etching technology for ARC formation was developed that would be less expensive and much faster than dry etching. Theoretical modeling revealed that dimple arrays should perform better than nipple arrays. A method of fabricating both dimple and nipple arrays having the same length was developed, and the dimple arrays performed better than the nipple arrays, in agreement with the modeling. The commercial viability of the technology is quite feasible, since the technology is scalable and inexpensive. This technology is also compatible with current industrial fabrication of solar cells. 


\section{CONTENTS}

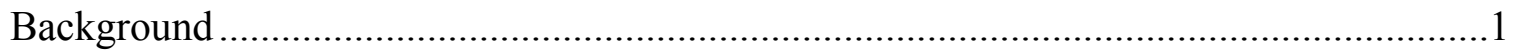

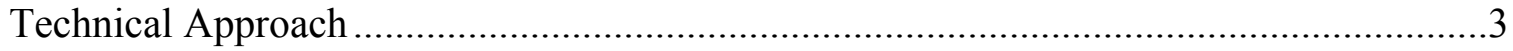

Experimental Methods and Results ............................................................................5

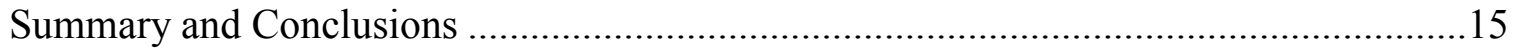

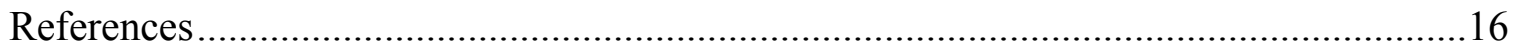

\section{FIGURES}

1. Solar spectrum and specular reflection of a solar cell having a conventional $\mathrm{SiN}_{\mathrm{x}}$ antireflective coating..

2. SEM image of a moth-eye corneal nipple array ………..................................................

3. Photograph and SEM images of silicon disk having spin-coated colloidal crystal-polymer nanocomposite ................................................................................................

4. SEM images of templated moth-eye ARC on crystalline silicon ..........................................4

5. Atomic force microscope image of templated polymer nipple array; experimental and modeled reflection from nipples as a function of nipple height ............................................

6. Surface reflectivity of ARC coated silicon exposed to various combinations of either temperature and humidity or Co-60 gamma irradiation (SRNL).........................................6

7. Surface reflectivity of ARC coated silicon exposed to various combinations of temperature

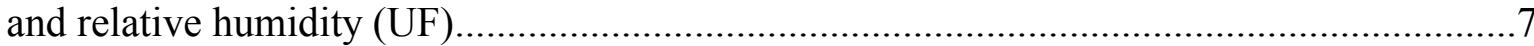

8. SEM images of ARC cross sections after exposure to temperature and humidity (UF) ........7

9. Surface reflectivity of ARC coated silicon after exposure to Co-60 gamma irradiation (UF).

10. SEM images of ARC coated silicon after exposure to various doses of Co-60 gamma irradiation.

11. SEM image of squarely-ordered domain ARC; comparison of optical reflection between square- and hexagonally- ordered ARCs....

12. SEM image of templated nano-sized ARC; reflectivity vs. wavelength (experiments and modeling) of templated ARC, commercial ARC on silicon solar cell, and uncoated silicon wafer

13. SEM images of ARC wet etched for several times.

14. Specular reflection versus wavelength for wet- and dry-etched silicon nanopillars 


\section{FIGURES (con't)}

15. AFM images of templated nipple- and dimple- arrays having similar structural parameters,

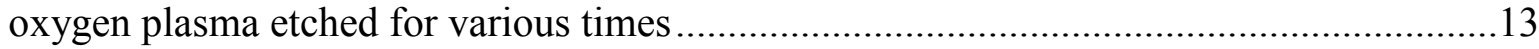

16. Specular reflection spectra from nipple- and dimple- arrays ............................................13

17. SEM image of templated polymer binary nipple-dimple ARC, and specular reflectivity ...14

\begin{tabular}{ll} 
& \multicolumn{1}{c}{ ABBREVIATIONS } \\
SRNL & Savannah River National Laboratory \\
UF & University of Florida, Department of Chemical Engineering \\
ARCs & Anti-reflection coatings \\
PECVD & Plasma enhanced chemical vapor deposition \\
TPV & Thermophotovoltaic \\
EBL & Electron beam lithography \\
IL & Interference lithography \\
ETPTA & Ethoxylated trimethylolpropane triacrylate \\
PMMA & Poly(methyl methacrylate) \\
RIE & Reactive ion etching \\
PDMS & Poly(dimethylsiloxane) \\
AFM & Atomic force microscope
\end{tabular}




\section{Background}

Solar cells (photovoltaics) produce electric power via conversion of the planet's most abundant and renewable energy input: sunlight. ${ }^{1-3}$ Crystalline silicon solar cells are among the most mature and efficient photovoltaic technology; in addition, silicon is the second most abundant element in the earth's crust. ${ }^{2}$ The production of photovoltaic panels is dominated by crystalline silicon solar cells with $98 \%$ of the market share. More specifically, $36 \%$ of the 2004 production is based on single crystal silicon, $58 \%$ on multi-crystalline silicon, and $4 \%$ on thin film amorphous silicon. ${ }^{3}$ Although silicon cells are the premier candidate for renewable and environmentally friendly solar energy conversion, high manufacturing cost has prevented such cells from economically competing with the combustion of fossil fuels as a source of electricity on a large scale. Currently, solar electricity is still 5-10 times more expensive than fossil-fuel and nuclear generated electricity due to high materials cost and low efficiency. ${ }^{1}$ If the materials for solar cells can be fabricated more economically and made more efficient in converting sunlight into usable power, the entire silicon solar cell market would be drastically affected. Given the current trend towards "green" energy, it is predicted that the global solar cell market could be worth somewhere between $\$ 18.6$ and $\$ 31.5$ billion by 2011 (solarbuzz.com).

Ideally, a solar cell should absorb all useful photons. However, due to the high refractive index of silicon, more than $35 \%$ of incident light is reflected back from the surface. ${ }^{4-6}$ Similar high reflection loss also limits the conversion efficiency of solar cells made by other inorganic semiconductors (e.g., concentrating $\mathrm{GaAs}$ cells and $\mathrm{GaSb}$ thermophotovoltaic cells) ${ }^{7-8}$ Anti-reflection coatings (ARCs) are therefore widely utilized to reduce the unwanted reflective losses and improve the conversion efficiencies of inorganic semiconductor solar cells. Quarter-wavelength silicon nitride $\left(\mathrm{SiN}_{\mathrm{x}}\right)$ films deposited by plasma enhanced chemical vapor deposition (PECVD) are the industrial standard for ARCs on crystalline silicon substrates. ${ }^{4}$ However, $\mathrm{SiN}_{\mathrm{x}}$ films are expensive to fabricate due to the high cost of chemical precursors and high vacuum processing conditions. Additionally, commercial $\mathrm{SiN}_{\mathrm{x}}$ ARCs can only suppress reflection efficiently at wavelengths around $600 \mathrm{~nm}$ (see the blue curve in Figure 1). ${ }^{2}$ The reflective loss is rapidly increased for nearinfrared and other visible wavelengths, which contain a large portion of the incident solar energy (see the solar spectrum in Figure 1). Moreover, because of the mismatch of the thermal expansion coefficient between the substrate and the anti-reflection materials, the thermal stability of the vacuum-deposited ARCs is usually poor. This greatly hampers the performance of solar cells operating in extreme conditions (e.g., outer space environment), and concentrating solar cells and thermophotovoltaic (TPV) cells where the surface temperature of

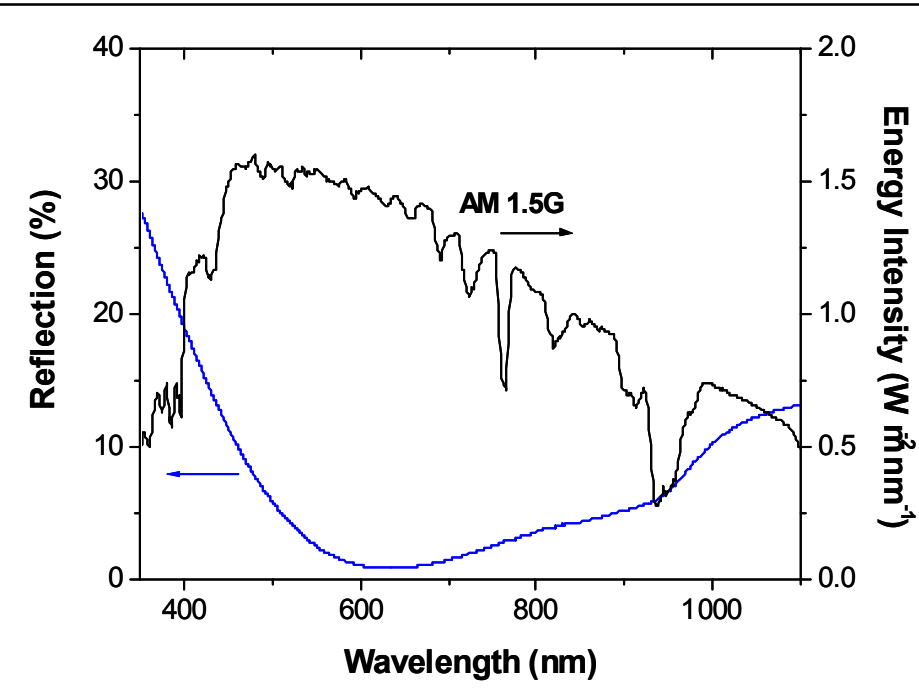

Figure 1. Standard $A M 1.5 \mathrm{G}$ solar spectrum and the specular reflection from a Plastecs single-crystal silicon solar cell with $\mathrm{SiN}_{\mathrm{x}}$ anti-reflection coating.

these cells is higher than that of a conventional cell. ${ }^{7-8}$ Therefore, to lower manufacturing costs and increase conversion efficiency of solar cells, it is highly desirable to develop inexpensive nanofabrication techniques that enable large-scale production of broadband ARCs with high coating stability and durability for reducing reflection over a broad range of wavelengths and angles of incidence. 
When the refractive index of the anti-reflection coating, $n_{c}$, is equivalent to the square root of the product of the indices of the surrounding medium, $n_{0}$, and the substrate, $n_{s}$, that is, $n_{c}=\left(n_{s} n_{0}\right)^{0.5}$, reflections are suppressed at wavelengths near the quarter-wavelength optical thickness. ${ }^{9}$ Consequently, $n_{c}$ must be between 1.2 and 1.3 for efficient ARCs to be suitable for glass and polymer substrates, while a higher $n(\sim$ 2.0 ) is needed for crystalline silicon. To achieve the low index of refraction requirement for transparent substrates, one solution is to use appropriately designed nanoporous materials, such as phase-separated polymers ${ }^{10-12}$ and nanoparticle and polyelectrolyte multilayers. ${ }^{13-17}$ Such materials can possess a very low effective $n$, due to the introduction of air, which has a value of $n=1$. For crystalline silicon substrates, quarter-wavelength $\mathrm{SiN}_{\mathrm{x}}$ films deposited by PECVD satisfy the above $n$ requirement and are the industrial standard for ARCs on silicon solar cells. ${ }^{4}$ Commercial $\mathrm{SiN}_{\mathrm{x}}$ ARCs are usually designed to present the reflectance minimum at around $600 \mathrm{~nm}$ where the flux of photons is maximal in the solar spectrum, ${ }^{2}$ while at other wavelengths, reflection significantly increases (Figure 1).

To achieve broadband anti-reflection coatings, it is further necessary to generate a graded refractive index such that $n_{c}$ close to the substrate surface matches $n_{s}$ and decreases to lower values further away from the surface. ${ }^{18-19}$ This idea is inspired by the natural grainy microstructures on the corneas of some nocturnal insects (e.g., moths) for the purpose of night camouflage. ${ }^{19-21}$ The outer surface of the corneal lenses of moths consists of a non-close-packed array of conical protuberances, termed corneal nipples, typically having less than 300 $\mathrm{nm}$ height and spacing (Figure 2). As the period of the nipple array is small compared to the wavelength of visible light, the optical properties of such a surface is governed by the effective medium theory ${ }^{12,19}$, and a graded transition of refractive index between air and the cornea is thus established. This leads to minimized reflection over a broad range of wavelengths and angles of incidence. ${ }^{19}$

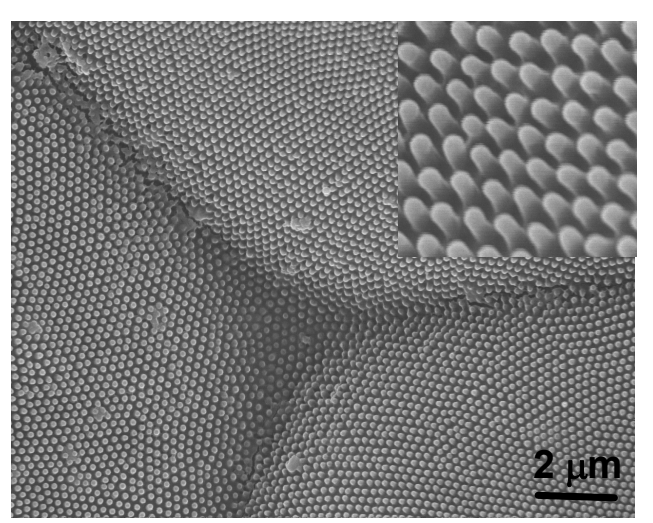

Figure 2. SEM images of a moth-eye corneal nipple array.

Top-down lithographic technologies, such as photolithography, ${ }^{5,12}$ electron-beam lithography (EBL), ${ }^{22}$ nanoimprint lithography, ${ }^{23}$ and interference lithography (IL), ${ }^{24-26}$ are commonly used to generate subwavelength nanostructured ARCs. However, these expensive techniques require sophisticated equipment and are limited by either low resolution or small sample size. Attaining high-throughput and large-area fabrication is a major challenge with these top-down technologies. Bottom-up techniques, such as colloidal self-assembly and templating nanofabrication, provide a much simpler and economical alternative to complex lithography in creating hexagonally ordered submicrometer-scale arrays. ${ }^{27-28}$ Unfortunately, traditional colloidal self-assembly and templating techniques suffer from low throughput and incompatibility with standard microfabrication, limiting the mass-production of practical ARCs. Furthermore, only limited, close-packed crystal structures are available through conventional colloidal self-assemblies, whereas non-close-packed structures are needed to mimic the natural moth eyes.

To resolve the scalability and compatibility issues of the current bottom-up approaches, an inexpensive and scalable spin-coating technology has been developed for manufacturing nanostructured broadband ARCs with greatly improved anti-reflection property than the PECVD-deposited $\mathrm{SiN}_{\mathrm{x}}$ coating at the University of Florida. ${ }^{29-39}$ This novel technology combines the simplicity and cost benefits of bottom-up colloidal self-assembly with the scalability and compatibility of standard top-down microfabrication. Although the capability of this nanofabrication technology in creating large-area broadband nanostructured ARCs has been demonstrated, ${ }^{30-38}$ significant issues need to be addressed before the 
ultimate implementation in practical solar cells. A systematic investigation of the structure-property relationships, which will greatly facilitate the rational design and fabrication of efficient moth-eye ARCs on a larger scale, has not yet been conducted. Moreover, the aging characteristics and ability to withstand typical environmental conditions (radiation exposure, heat fluctuations, humidity, etc) of a commercial solar cell with the proposed ARC, has not yet been investigated, which is crucial in the evaluation of market readiness. The work described in this report involved exposing the new ARC to either heat and humidity or gamma irradiation and subsequently measuring the properties of the ARC to determine whether the desirable properties of the ARC are retained after the exposure.

\section{$\underline{\text { Technical Approach }}$}

\section{Large-Scale Assembly of Colloidal Particles on Crystalline Silicon Wafers}

Colloidal particles are assembled on crystalline silicon wafers using the spin-coating technology developed at the University of Florida. ${ }^{29,40-44}$ Spin-coating enables rapid production of colloidal crystals with remarkably large domain sizes and unusual non-close-packed surface structures (Figure 3). ${ }^{29,40-44}$ The methodology is based on shear-aligning concentrated colloidal suspensions. Monodispersed silica colloids with diameters ranging from $\sim 70 \mathrm{~nm}$ to $\sim 2 \mu \mathrm{m}$, which can be inexpensively synthesized by the wellestablished Stöber method, ${ }^{45}$ are dispersed in non-volatile ethoxylated trimethylolpropane triacrylate (ETPTA) monomers. The particle volume fraction of the suspension is $\sim 20 \%$. The suspensions are then spincoated at speeds ranging from 200 to $8000 \mathrm{rpm}$ using standard spin-coating equipment. Silicon wafers, glass slides, and poly(methyl methacrylate) (PMMA) plates, among many others have successfully been used as substrates. ${ }^{29}$ Formation of wafer-scale colloidal crystals occurs within seconds as indicated by the appearance of a striking diffraction star with six arms (Figure 3A); this pattern is characteristic of longrange hexagonal ordering. . $^{29,46}$ The monomers rapidly polymerize by exposure to

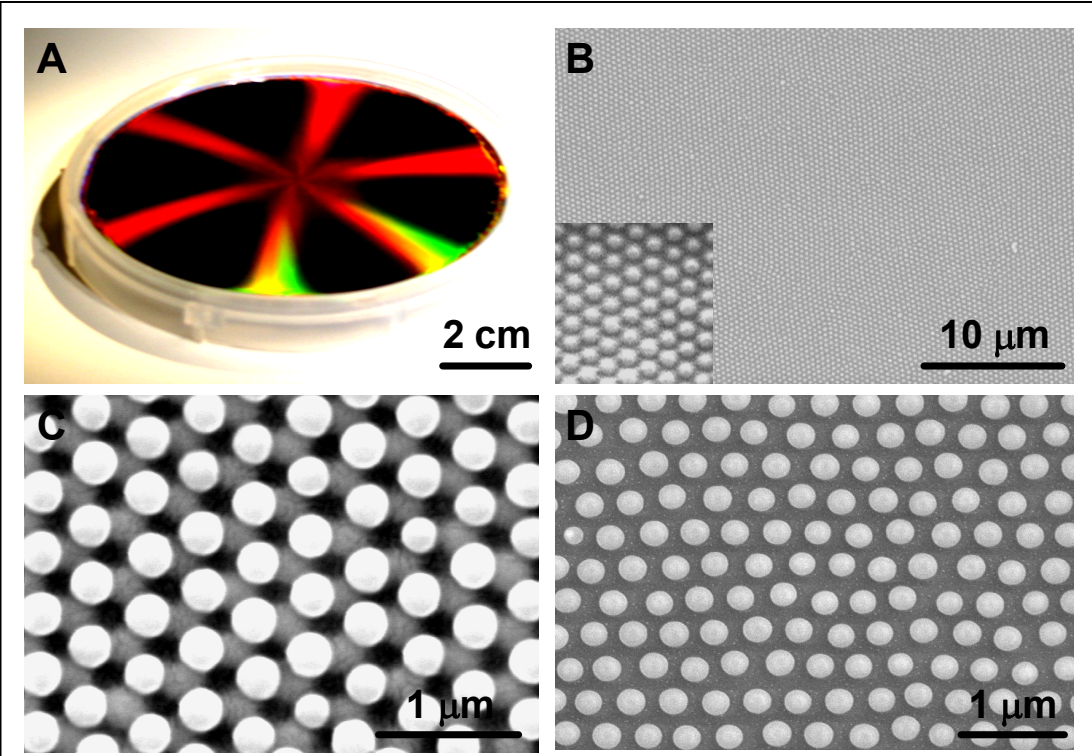

Figure 3. (A) Photograph and (B) SEM image of a 4-inch, 3-D ordered colloidal crystal-polymer nanocomposite made by spincoating. Nonclose-packed multilayer (C) and monolayer (D) colloidal crystals after removing the polymer matrix of spin-coated nanocomposites.

ultraviolet radiation to form ordered nanocomposites (Figure 3B). The polymer matrix can finally be removed by oxygen plasma etching to release the embedded colloidal crystals (Figure 3C and 3D). The spin-coating technique enables mass-fabrication of wafer-scale (up to 12 in. diameter) colloidal crystals, which is a length scale nearly three-orders of magnitude larger than currently available through other methods. ${ }^{47}$ Additionally, the entire crystal is formed within minutes, as compared to days or even weeks needed to produce a centimeter-size crystal using other self-assembly techniques. Most important, the spin-coating technique is compatible with the current industrial production processes of crystalline silicon solar cells. 


\section{Templated Fabrication of Moth-Eye ARCs on Crystalline Silicon Wafers}

Using the spin-coated monolayer colloidal crystals (Figure 3D) as structural templates, an inexpensive and scalable bottom-up technology for fabricating broadband ARCs on crystalline silicon wafers has been developed. ${ }^{31,35}$ The nonclosepacked silica particles can be used as etching masks during a chlorine Reactive Ion Etching (RIE) process $(5 \mathrm{mT}$ Torr pressure, 20 SCCM chlorine flow rate, and $80 \mathrm{~W})$ to pattern arrays of subwavelength silicon pillars directly on the substrate (Figure 4). As the etching rate of silica is much lower than that of silicon under above RIE conditions, silica particles protect silicon immediately underneath them from being etched, resulting in the formation of pillar arrays directly on silicon surface (Figure 4B). Once the silicon pillars are deep enough, the templating silica

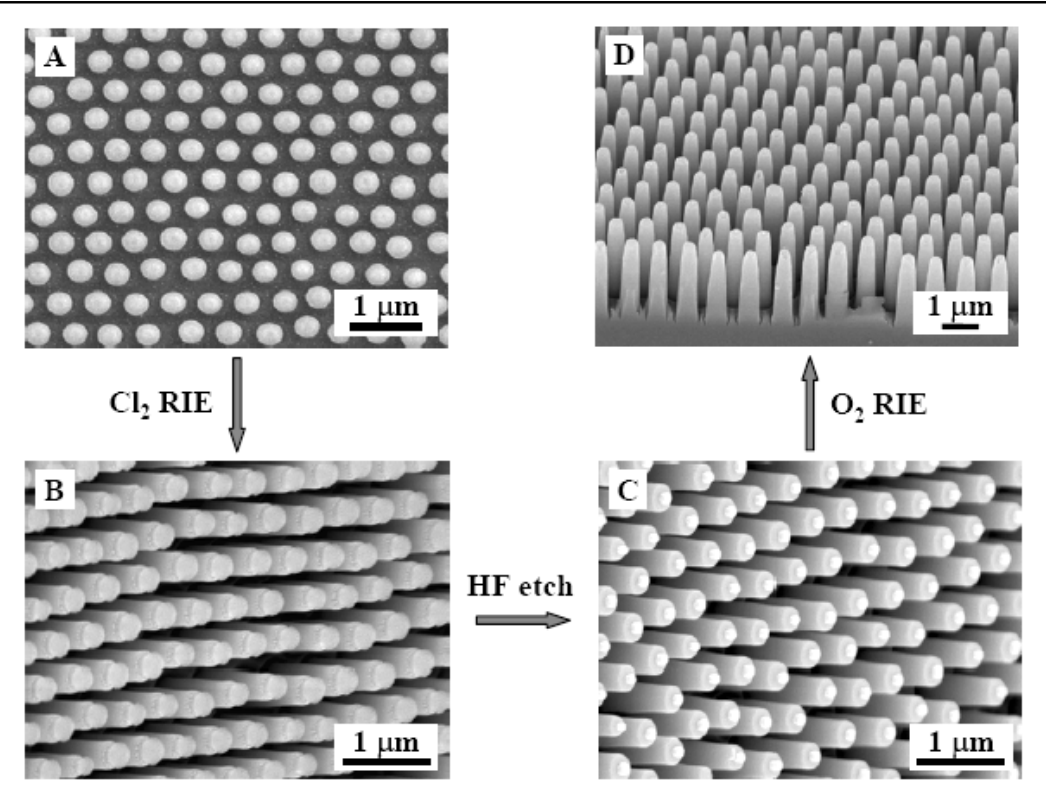

Figure 4. Templated fabrication of moth-eye antireflection coatings on crystalline silicon by using self-assembled colloidal silica particles as etching masks. spheres can be removed by dissolving them in a $2 \%$ hydrofluoric acid aqueous solution. Interestingly, arrays of "micro-candles" are observed, consisting of silicon columns as candle bodies and polymer dots as candle wicks (Figure 4C). The polymer dots are unetched residue of the thin polymer wetting layer $(\sim 100 \mathrm{~nm}$ thick) between the spin-coated colloidal monolayer and the silicon substrate. These dots can be easily removed by brief oxygen RIE to generate clean silicon pillar arrays (Figure 4D). The resulting pillar arrays exhibit excellent broadband anti-reflection properties and the reflectivity is below $2.5 \%$ for all wavelengths ranging from 400 to $1100 \mathrm{~nm}$. This is in sharp contrast to commercial $\mathrm{SiN}_{\mathrm{x}} \mathrm{ARCs}_{\mathrm{s}}$ that exhibit low reflection only at wavelengths around $600 \mathrm{~nm}$ (see the blue curve in Figure 1).

\section{Templated Transparent Moth-Eye ARCs on Transparent Substrates}

Spin-coating also provides a simple and scalable platform for fabricating transparent polymer and sol-gel glass moth-eye ARCs on glass substrates. The periodic surface modulations of the shear-aligned nanocomposites (Figure 3B) can be easily transferred to a poly(dimethylsiloxane) (PDMS) master. Polymer ARCs with adjustable reflectivity can then be molded against the PDMS master by either thermal or photo-polymerization of a large variety of monomers on both planar and curved substrates (Figure 5A). The polymer matrix of the spin-coated nanocomposites can be plasma-etched to adjust the height of the protruded portions of silica spheres, resulting in good control over the depth of replicated nipples and the corresponding specular reflection (Figure 5B). The templated hemispherical nipple array (blue solid curve in Figure 5B) exhibits significantly lower reflection than a flat polymer (black solid curve) for the whole visible spectrum. Figure 5B also illustrates the reasonably good agreement between the experimental spectra and the optical simulation based on a thin-film multilayer model. 
SRNL-STI-2011-00094
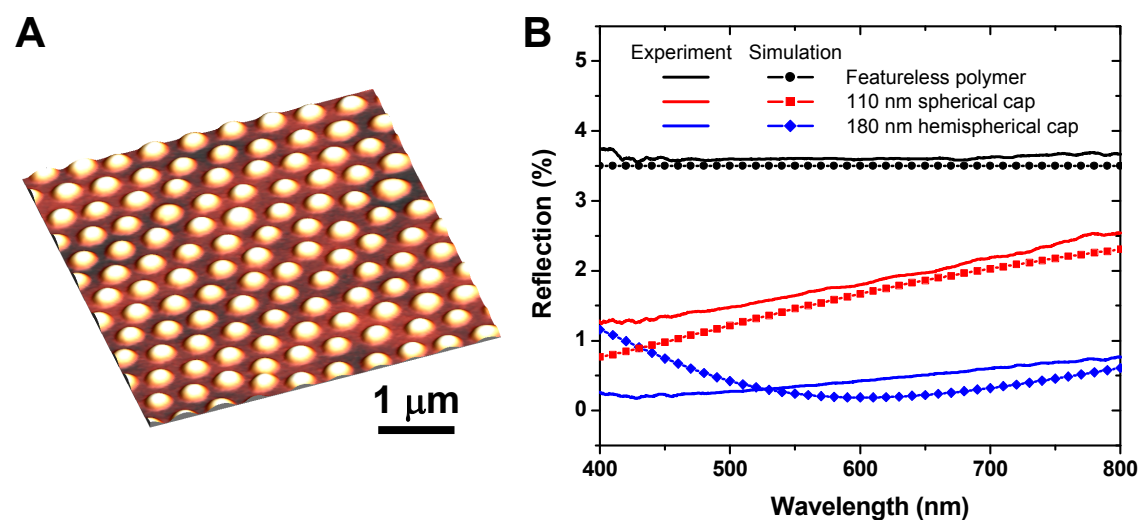

Figure 5. (A) AFM image of a templated polymer nipple array. (B) Experiment and modeled reflection from nipples with different heights.

\section{Experimental Methods and Results}

\section{Exposure to Heat/Humidity and Gamma Irradiation at SRNL; SRNL Reflectivity Measurements}

ARC coated samples fabricated at the University of Florida were exposed to two controlled environments: 1) elevated temperature and humidity; and 2) gamma irradiation. The elevated temperature and humidity environment exposure demonstrated whether the new coating could withstand the temperature and humidity that solar cells are exposed to in operation on earth, and the gamma irradiation will demonstrate the robustness of the coating for use in the radiation environments such as the International Space Station, and other space satellites.

Samples were cut at SRNL from a silicon wafer treated with the ARC at the University of Florida. Samples were exposed at SRNL for varying lengths of time for up to 60 days at either $40{ }^{\circ} \mathrm{C}$ and $95 \%$ relative humidity $(\mathrm{RH}), 60{ }^{\circ} \mathrm{C}$ and $80 \% \mathrm{RH}$, or $80{ }^{\circ} \mathrm{C}$ and $40 \% \mathrm{RH}$ in a controlled temperature/humidity oven (Cincinnati Sub-Zero ZH-32-3-3-H/WC Temperature/Humidity Test Chamber, Model Z-32). Four samples were exposed to a cobalt-60 source (J. L. Shepherd, Model 109) in air at ambient temperature; the total doses were $5 * 10^{7} \mathrm{Rad}, 1 * 10^{8} \mathrm{Rad}, 2.5^{*} 10^{8} \mathrm{Rad}$, and $5^{*} 10^{8}$ Rad. Reflectivity of the temperature/humidity and gamma exposed samples was measured as a function of frequency at SRNL. These reflectivity measurements were made using an Ocean Optics USB 2000 with optic reflective probe 727-733-2447 and R400-7-Vis/NIR optical fibers. An Ocean Optics LSI-1 tungsten halogen lamp was used as the light source and reflectivity was measured against Ocean Optic Hi and Low Reflective standards, PN: STAN-SSH and STAN-SSL. The spectrometer was operated using Ocean Optic OOIBase32, versions 2.0.1.4 . The reflectivity is shown as a function of wavelength in Figure 6, for both radiation dose exposed and the \% relative humidity-temperature-time exposed samples. Also shown in Figure 6 is the reflectivity of uncoated silicon, showing the significant reduction in reflectivity caused by the anti-reflective coating at all wavelengths measured. None of the environments tested significantly deteriorated the performance of the coating at any wavelength. This is the main result of this study and demonstrates the robustness of the new coating and its anti-reflective properties. 


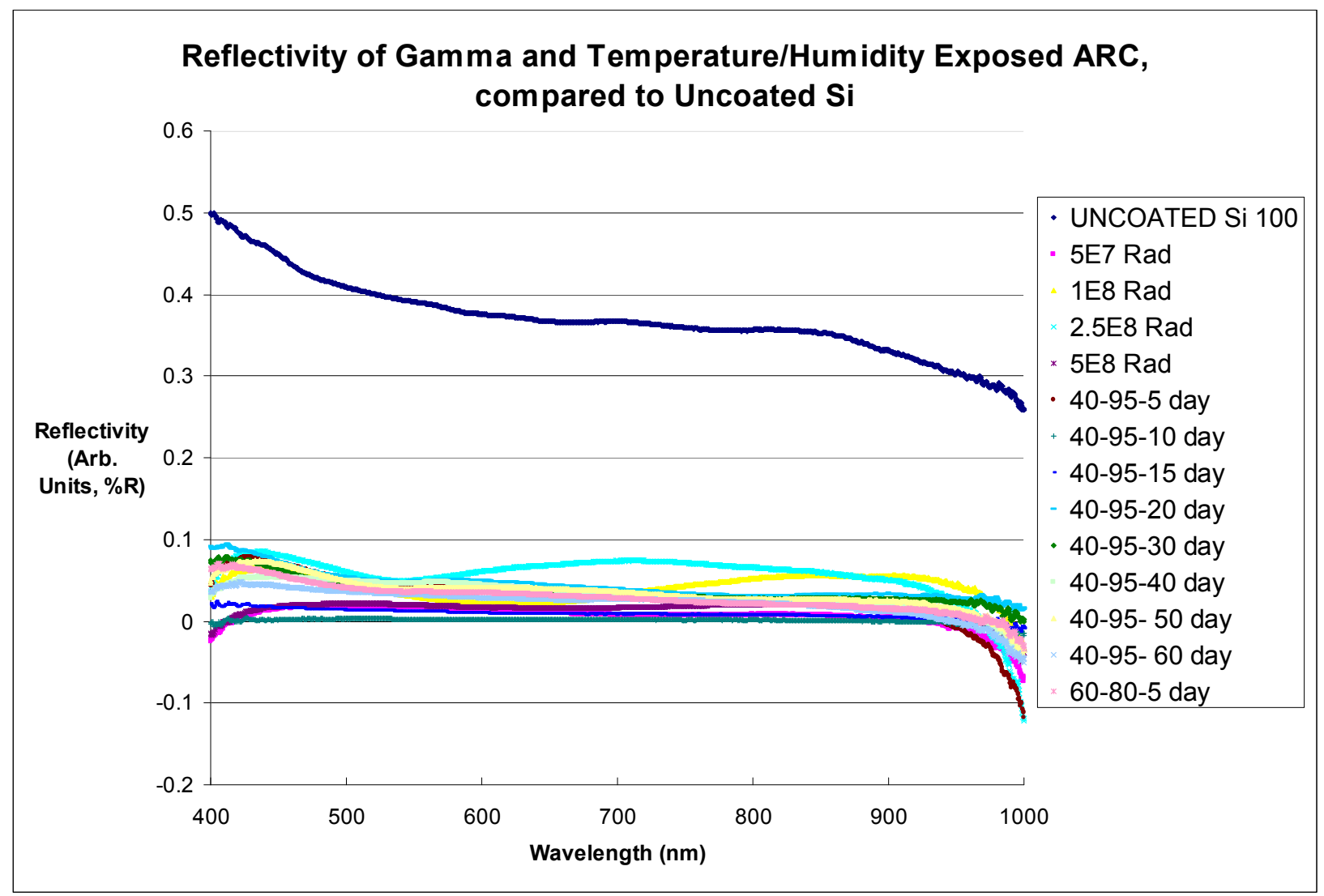

Figure 6. Surface reflectivity (SRNL) of samples with ARC exposed to various combinations of either temperature and humidity or gamma irradiation.

\section{Further Characterization of Exposed Samples and Development of Optimal Moth-Eye ARCs at UF}

\section{Characterization of Environment-Aged Moth-Eye ARCs}

A HR4000 High Resolution Fiber Optic UV-Vis spectrometer and a NIR-512 spectrometer (both from Ocean Optics) with reflection probes were used for reflectance measurements. A calibrated halogen light source was used to illuminate the sample and the spectrometers can scan wavelengths from 350-1650 $\mathrm{nm}$. The beam spot size is about $3 \mathrm{~mm}$ on the sample surface. Measurements are performed at normal incidence and the cone angle of collection is less than $5^{\circ}$. Absolute reflectivity is obtained as ratio of the sample spectrum and the reference spectrum. The reference spectrum is the optical density obtained from an aluminum-sputtered (1000 $\mathrm{nm}$ thickness) silicon wafer. Final value of absolute reflectivity is the average of several measurements obtained from different spots on the sample surface. The resulting reflectivity is calibrated using an Ocean Optics STAN-SSL low-reflectivity specular reflectance standard for templated pillar arrays and glass slides and a STAN-SSH High-reflectivity Specular Reflectance Standard for flat silicon wafers. 
The light reflectivity and surface microstructure of the exposed material samples were also characterized at the University of Florida. The elevated temperature and humidity environment exposure will demonstrate whether the new coating can withstand the temperature and humidity solar cells are exposed to in operation on earth, and the gamma irradiation will demonstrate the robustness of the coating for use in the radiation environments such as the International Space Station, and other space satellites. A silicon wafer was treated with the coating at UF, and samples were cut from it at SRNL. Samples were exposed for varying lengths of time for up to 60 days at either $40{ }^{\circ} \mathrm{C}-95 \%$ relative humidity $(\mathrm{RH}), 60{ }^{\circ} \mathrm{C}-80 \% \mathrm{RH}$, or $80{ }^{\circ} \mathrm{C}-40 \% \mathrm{RH}$ in a controlled temperature/humidity oven (above). Reflectivity of these exposed samples was measured as a function of frequency first at SRNL (above), then again at UF (Figure 7). Measurements at both laboratories were consistent- none of the environments tested to date significantly deteriorated the performance of the coating at visible wavelength- this is an important result of this study and demonstrates the robustness of the new coating and its anti-reflective properties.
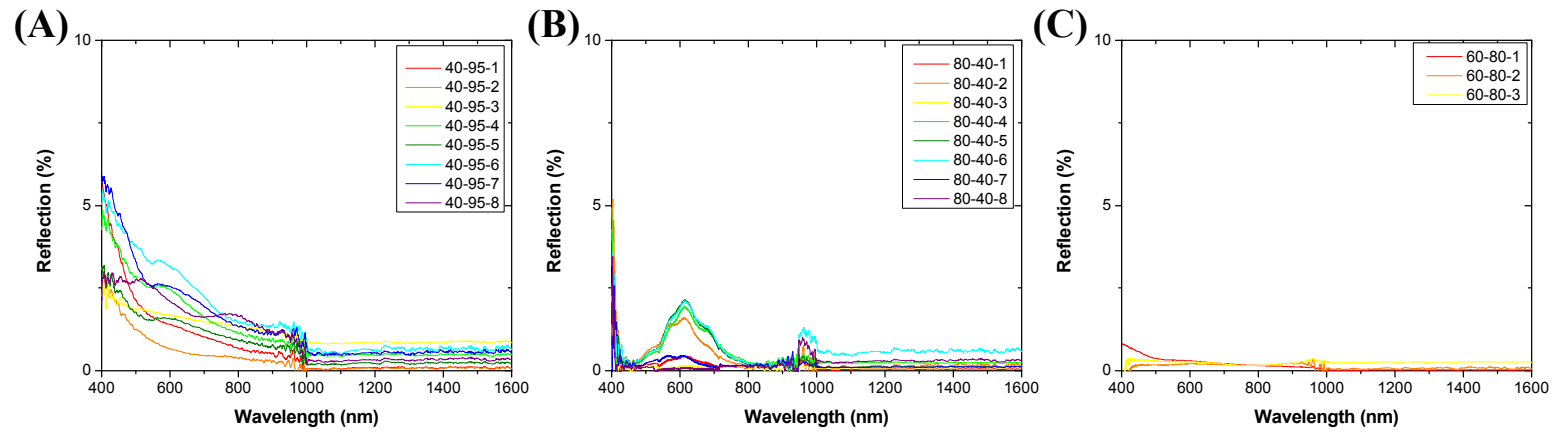

Figure 7. Normal incidence optical reflection spectra obtained from templated ARCs exposed to various aging environments (UF). (A) $40{ }^{\circ} \mathrm{C}-95 \% \mathrm{RH}$, (B) $80{ }^{\circ} \mathrm{C}-40 \% \mathrm{RH},(\mathrm{C}) 60{ }^{\circ} \mathrm{C}-80 \% \mathrm{RH}$

Scanning electron microscopy (SEM) at University of Florida is carried out on a JEOL 6335F FEG-SEM. A thin layer of gold is sputtered onto the samples prior to imaging. Both top-view and cross-sectionalview SEM images are obtained.

SEM examination of the coatings revealed no significant change of the morphology of the coating by thermal/humidity exposure (Figure 8):
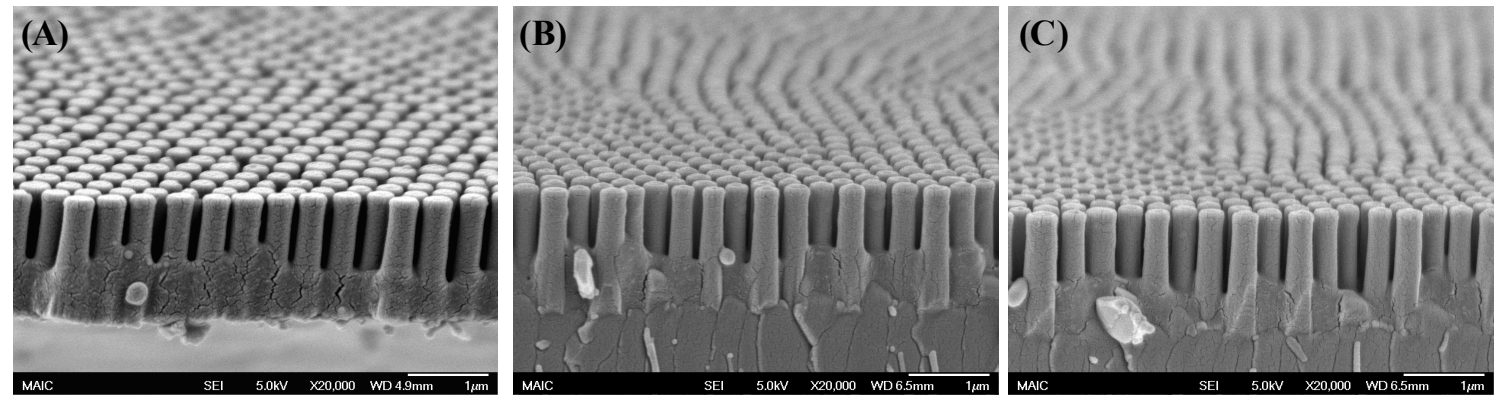

Figure 8. SEM images of moth-eye ARCs aged at different conditions. (A) $40{ }^{\circ} \mathrm{C}-95 \% \mathrm{RH}$, (B) $80{ }^{\circ} \mathrm{C}$ $40 \% \mathrm{RH},(\mathrm{C}) 60{ }^{\circ} \mathrm{C}-80 \% \mathrm{RH}$

The samples exposed to gamma irradiation at SRNL were also tested at the UF. The effect of gamma irradiation is to actually reduce the reflectivity- the coating is more anti-reflective, (that is, better) as a function of dose at low wavelengths (Figure 9). 


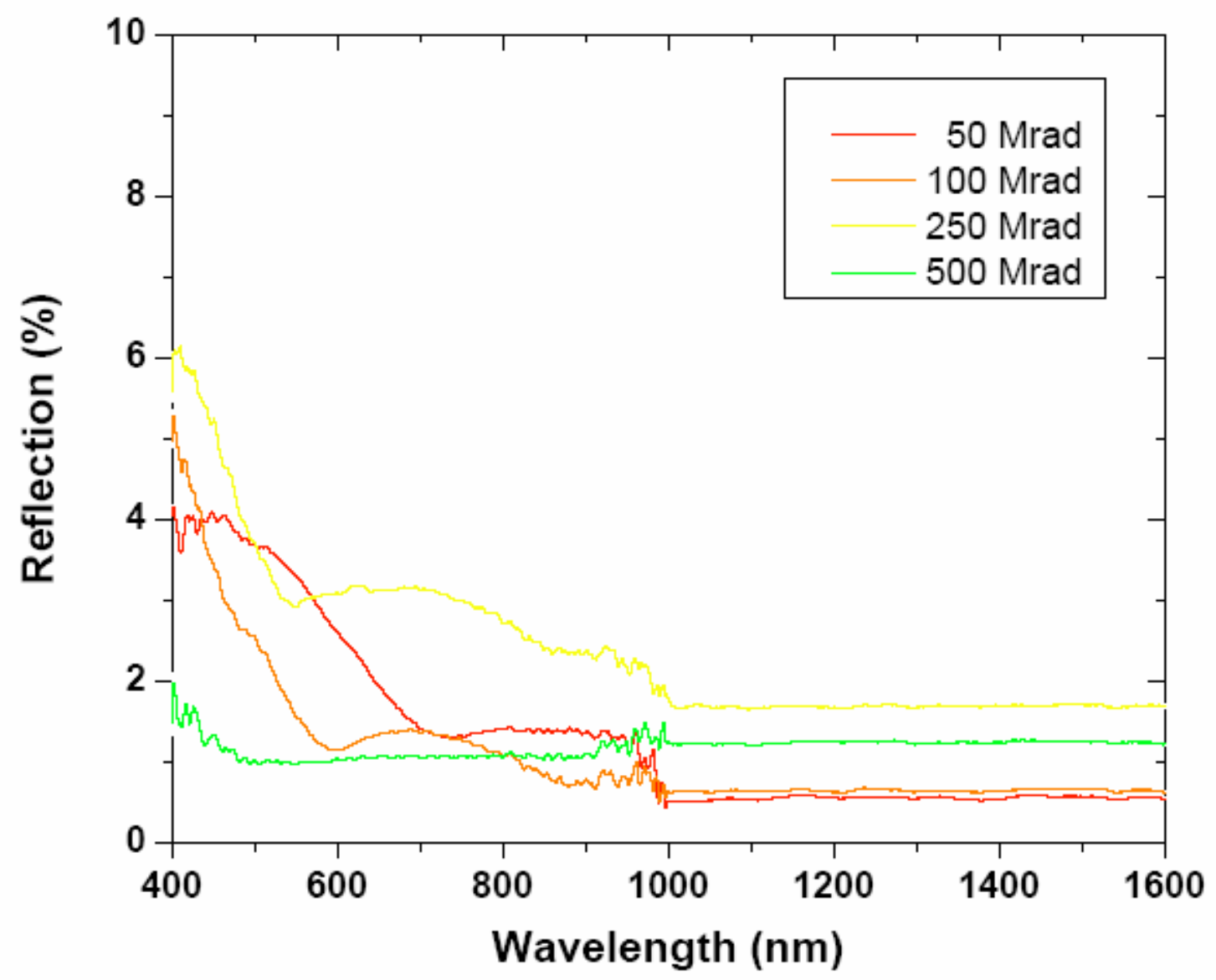

Figure 9. Reflectivity as a function of wavelength for gamma irradiated samples (measured at UF). Dose as noted.

SEM examination at UF revealed the surface morphology is unaffected by gamma radiation exposure (Figure 10). 

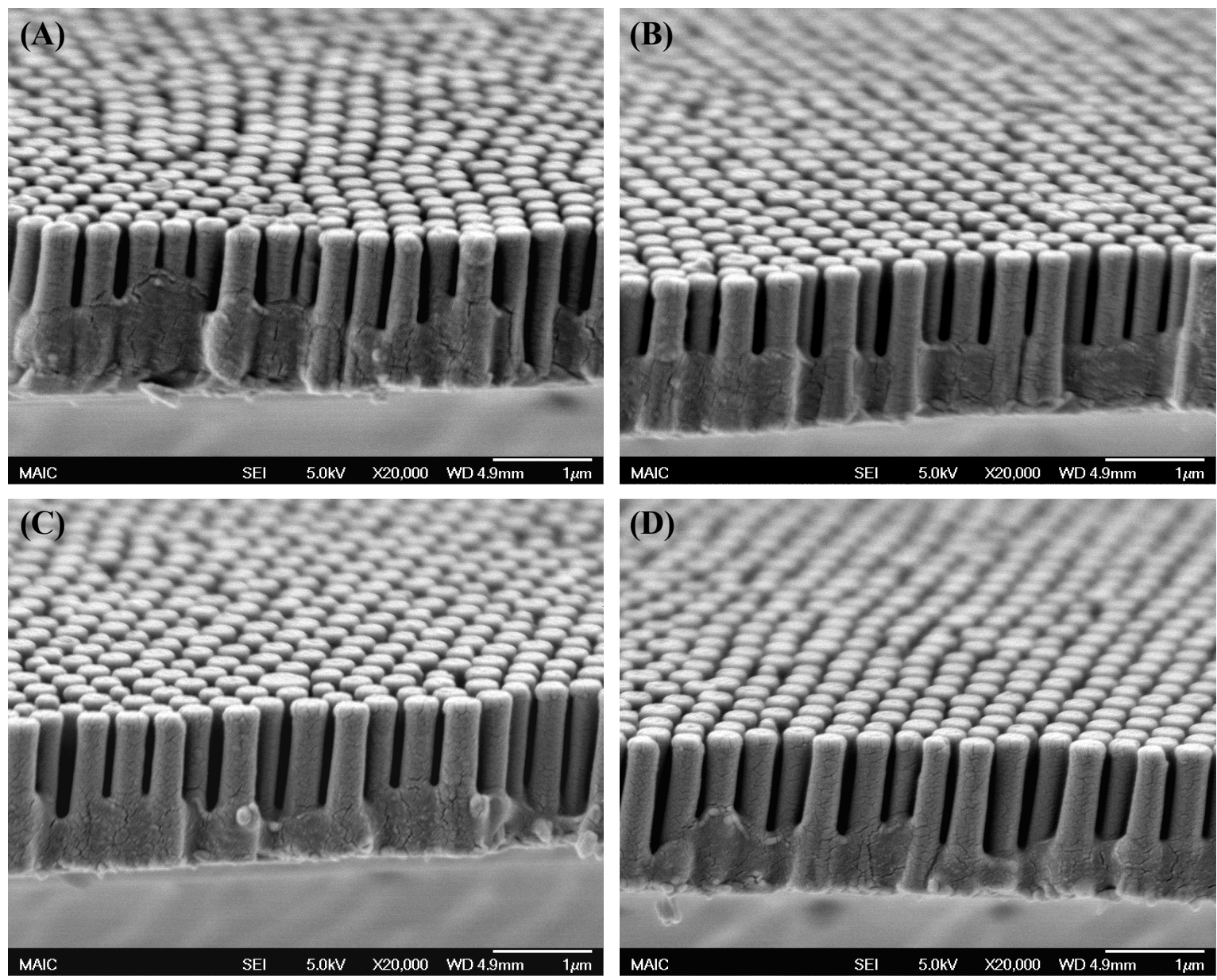

Figure 10. SEM images of moth-eye type ARCs exposed to gamma radiation at various doses. (A) 50 Mrad, (B) $100 \mathrm{Mrad}$, (C) $250 \mathrm{Mrad}$, (D) $500 \mathrm{Mrad}$.

The UF team also exposed the templated silicon moth-eye ARCs to very high intensity ultraviolet light (1200 Watt-Seconds UV radiation produced by a Xenon RC-801 system using a Type B lamp (broad band spectrum cut off at $240 \mathrm{~nm})$ for one day and high intensity $(2.0 \mathrm{~W})$ continuous $532 \mathrm{~nm}$ laser for 2 weeks. Similar to the results as shown in Figures 6, 7, 8, 9, and 10, SEM images and normal-incidence reflection measurements demonstrate that the effect of $\mathrm{UV}$ and visible radiation on the antireflection performance of the templated ARCs is very small.

\section{Development of Optimized Moth-Eye ARCs}

A systematic experimental and theoretical program to optimize moth-eye ARCs on singlecrystalline silicon substrates was performed at UF. First, the effect of the crystalline domain size and structure on the antireflection properties of moth-eye ARCs was evaluated. The crystalline domain size was found to play only a minor role in determining the antireflective performance. The square-ordered moth-eye ARCs exhibit better antireflective properties than hexagonallyordered ARC gratings. Figure 11A shows an SEM image of a templated moth-eye ARC with long-range square ordering. Figure 11B compares the antireflection performance between a hexagonally ordered moth-eye grating and a square array with the same crystalline parameters 
(size, spacing, depth, etc). It is apparent that the square array is a better antireflection structure. In addition, the optical reflection is below $2 \%$ for most of the visible spectrum.
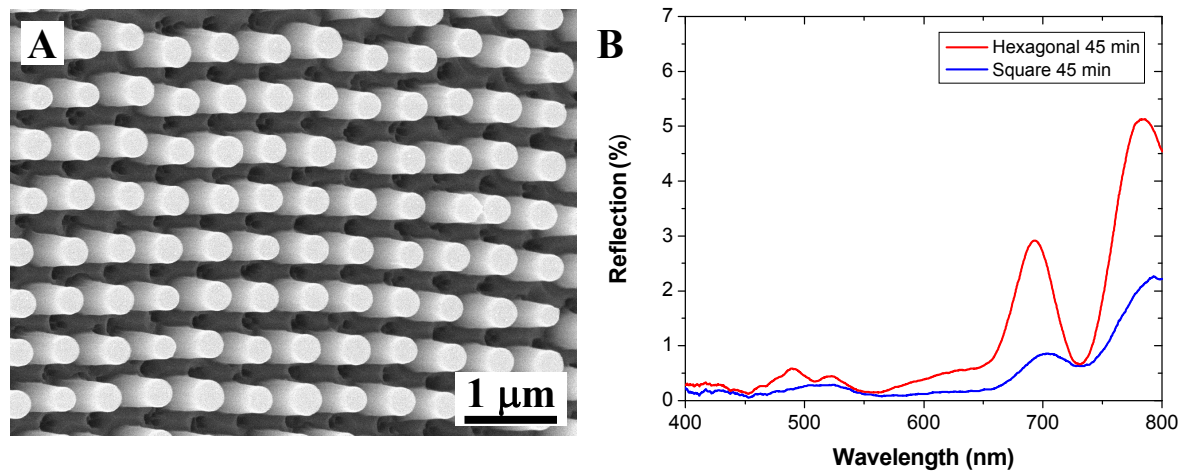

Figure 11. (A) SEM image of a templated moth-eye antireflection grating with squarely ordered domains. (B) Comparison of normal-incidence optical reflection between a squarely and a hexagonally ordered ARCs.

The size effect of the templated nanopillars on the antireflection performance of moth-eye ARCs was also evaluated. We have demonstrated that $\sim 70 \mathrm{~nm}$ silica particles can be assembled over wafer-scale areas by using the spin-coating technology (Figure 12A). Nano-scale moth-eye ARCs have been successfully fabricated by using chlorine reactive ion etching. The resulting nanostructured pillar arrays exhibit excellent broadband antireflective and superhydrophobic properties, promising for developing self-cleaning antireflection coatings for crystalline silicon solar cells. Another big advantage of the nano-scale ARCs is that the Bragg diffraction of visible light by the traditional submicrometer-scale ARCs can be eliminated. This will further improve the antireflection properties of moth-eye ARCs.
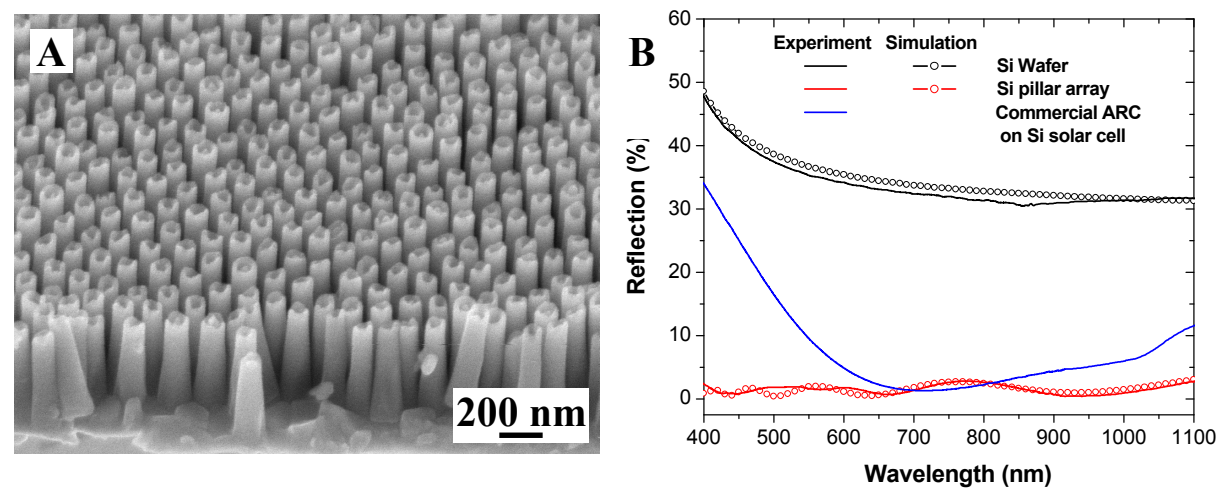

Figure 12. (A) Templated nano-sized moth-eye ARC. (B) Normal-incidence optical reflection and numerical simulation from the templated ARC, a commercial ARC on a Si solar cell, and a bare Si wafer.

As shown by our theoretical simulation, the depth of the templated nanopillars also plays a critical role in determining the resulting antireflection properties of moth-eye ARCs. A sufficient optical depth is required to achieve good antireflection properties. However, the dry etchingbased templating technology as described above requires long etching time (usually tens of 
minutes) to obtain nanopillars with high aspect ratio. This could limit the throughput and cost of the resulting antireflection coatings. We therefore developed a wet etching technology which is less inexpensive and much faster than dry etching. The spin-coated silica particles can be used as structural template to create gold (or silver, $\sim 30 \mathrm{~nm}$ thick) nanohole arrays on silicon. The metallized silicon wafer is then immersed in an aqueous solution of hydrofluoric acid and hydrogen peroxide $\left(\mathrm{HF} / \mathrm{H}_{2} \mathrm{O}_{2} / \mathrm{H}_{2} \mathrm{O}, \mathrm{v} / \mathrm{v} / \mathrm{v}=10 / 5 / 35\right)$ at room temperature. The silicon surface that comes in contact with gold is catalytically oxidized by gold and the resulting oxide is removed by hydrofluoric acid, leading to the formation of highly ordered SiNWs whose diameter is predefined by the size of the nanohole, while the length is determined by the etching time. Figure 13A-C shows the side-view SEM images of SiNWs templated from the same array of polymer posts but wet-etched for different time. From Figure 13D, it is evident that the length of silicon nanopillars increases with longer etching time, while the diameter decreases only slightly, indicating anisotropic characteristics of the metal-assisted wet etching. The silicon substrate with the wet-etched high-AR SiNWs is much darker than the bare wafer. Nanopillars with higher aspect ratio achieved by wet-etching have larger optical depth and thus facilitate more efficient suppression of specular reflection than dry-etched pillars (Figure 14).

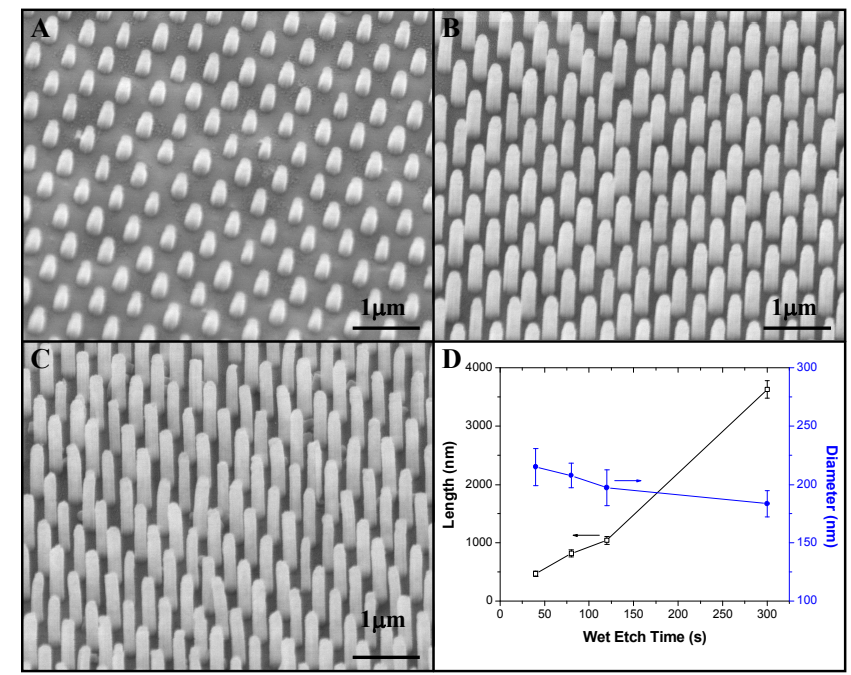

Figure 13. (A (A-C) Tilted-view $\left(40^{\circ}\right)$ SEM images of SiNWs templated from the same polymer post array as shown in Figure 1D and wet-etched for 40, 80, and $120 \mathrm{~s}$, respectively. (D) Dependence of the length and diameter of templated SiNWs on wet-etch time. 


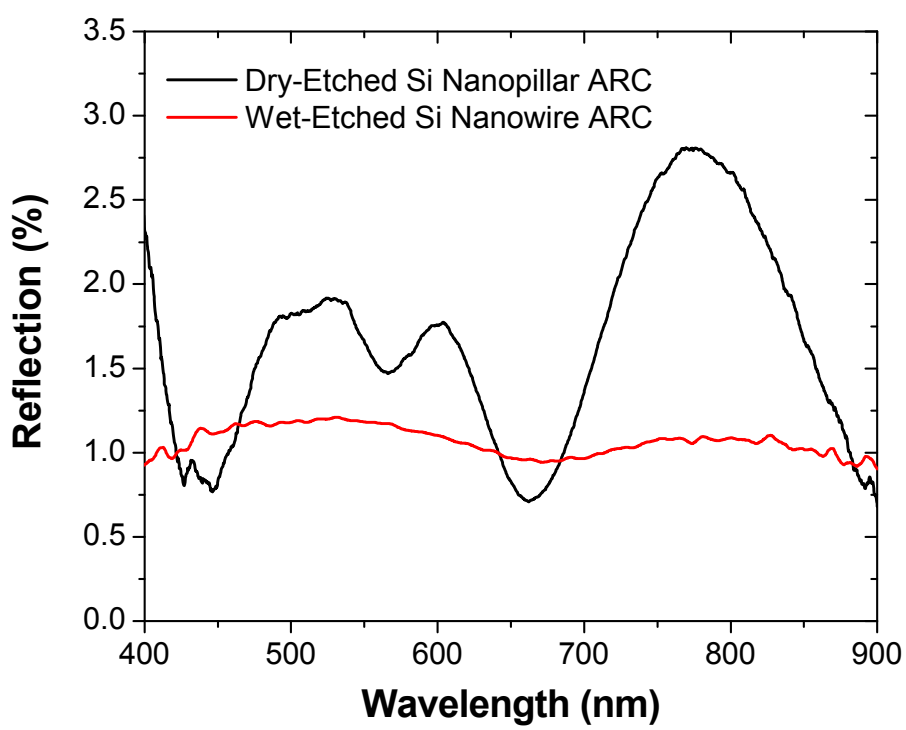

Figure 14. Comparison of the specular optical reflection at normal incidence from wet-etched ( $5 \mathrm{~min}$ ) and dry-etched (40 min) Si nanopillars.

Besides silicon moth-eye ARCs, we have also conducted systematic studies on the optimization of transparent (e.g., sol-gel glass and polymer) moth-eye ARCs for reducing optical reflection from top glass encapsulation layer of a solar cell panel. Our theoretical calculations show that subwavelength dimple arrays are better antireflection structure than nature-inspired moth-eye nipple arrays. To verify this important prediction for creating efficient ARCs, we have conducted systematic experimental investigations. As we know from the optimization of silicon moth-eye ARCs, the depth of the moth-eye structure plays a crucial role in determining the antireflective properties. To fairly compare the dimple and nipple structures, the depths of these features needs to be the same. To accomplish this, we have developed a two-step soft-lithography approach to create dimple and nipple arrays with the same depth. We first used the top-layer of the spin-coated colloidal arrays as the first-generation template to create a polydimethylsiloxane (PDMS) mold. The depth of the PDMS mold is the same as the protrusion depth of the silica spheres embedded in the colloidal arrays. We then used this PDMS mold to create a polymer nipple array on a glass substrate by a soft-lithography-like templating technique. To create a polymer dimple array with the same depth, we used the PDMS mold as a second-generation template to create another hard PDMS mold with the inverted structure. We finally used the hard PDMS mold to create polymer dimple arrays with the same depth as that of the nipple arrays. Figure 15 shows the atomic force microscope (AFM) images of templated nipple and dimple arrays. It is apparent that these structures have very similar structural parameters (e.g., feature size, depth, separation etc.). This ensures a fair comparison of the antireflective properties between a nipple and dimple structure. Figure 16 shows the comparison of the normal-incidence optical reflection from the nipple and dimple arrays. It is evident that the dimple arrays show better antireflection performance than that of the nipple arrays with the same structural parameters, agreeing with our theoretical prediction. 

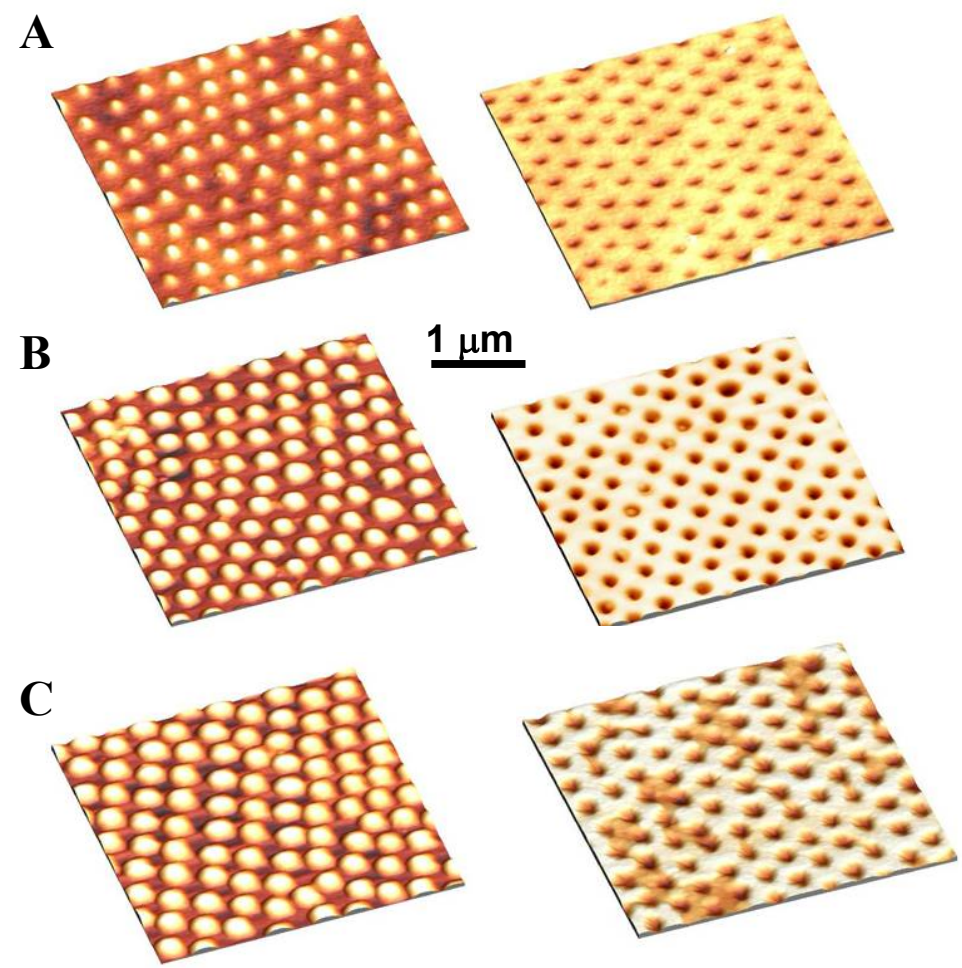

Figure 15. AFM images of templated nipple and dimple arrays with similar structural parameters. These samples were replicated from a spin-coated colloidal array after a certain time of oxygen plasma etching. (A) 0 s. (B) $30 \mathrm{~s}$. (C) $45 \mathrm{~s}$.

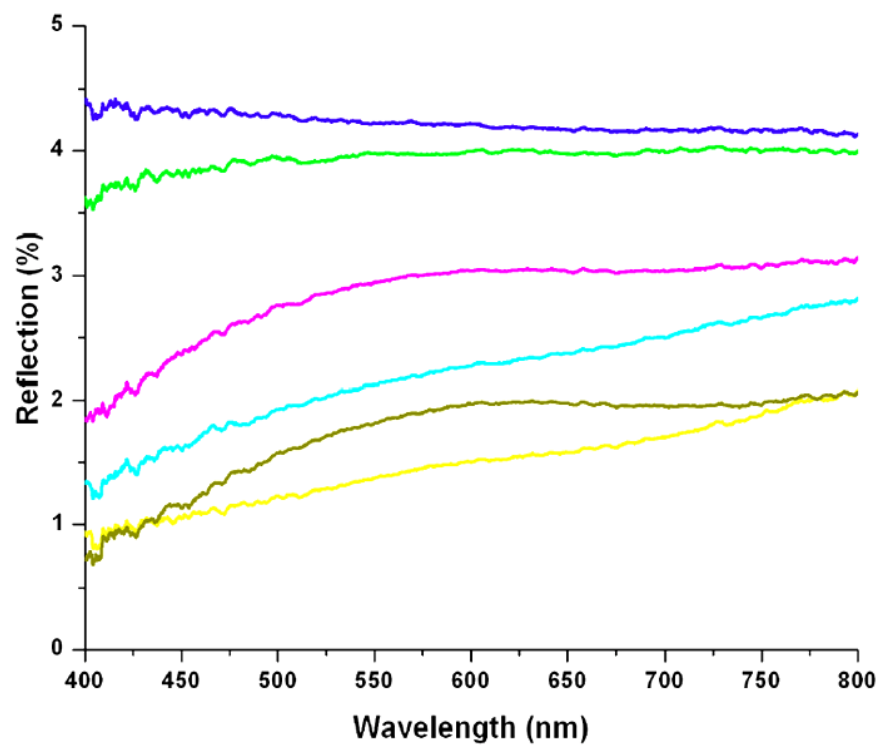

Figure 16. Normal-incidence optical reflection spectra from templated nipple and dimple arrays. These samples were prepared by replicating a colloidal array plasma-etched at different durations. (Blue) Nipple array, $0 \mathrm{~s}$ etching. (Green) Dimple array, $0 \mathrm{~s}$ etching. (Magenta) Nipple array, $30 \mathrm{~s}$ etching. (Cyan) 
Dimple array, $30 \mathrm{~s}$ etching. (Dark Yellow) Nipple array, $45 \mathrm{~s}$ etching. (Yellow) Dimple array, $45 \mathrm{~s}$ etching.

We have also evaluated antireflection performance of moth-eye ARCs with even complex microstructures. One example is dimple-nipple antireflection coatings, which are easily available by the spin-coatingbased templating technology. Double-layer, nonclose-packed colloidal crystals prepared by spin-coating are used as etching masks to define the resulting binary crystalline structures (Figure 17A). Contrary to conventional colloidal lithography, the polymer matrix between the self-assembled colloidal crystal is also been utilized as sacrificial template. The solid lines in Figure 17B show the experimental reflection spectra from a flat glass substrate and a glass slide covered with a polymer dimple-nipple ARC. For comparison with the new binary antireflective structure, the normal-incidence reflection from a glass slide coated with a unitary polymer hemispherical ARC is also shown (blue curve). The glass control sample exhibits $\sim 4 \%$ single-surface reflection, while the binary ARC coated glass slide displays $<0.5 \%$ reflectivity for the whole visible spectrum. It is also evident that the binary ARC shows improved antireflective performance than the unitary hemispherical ARC for most of the visible wavelengths.
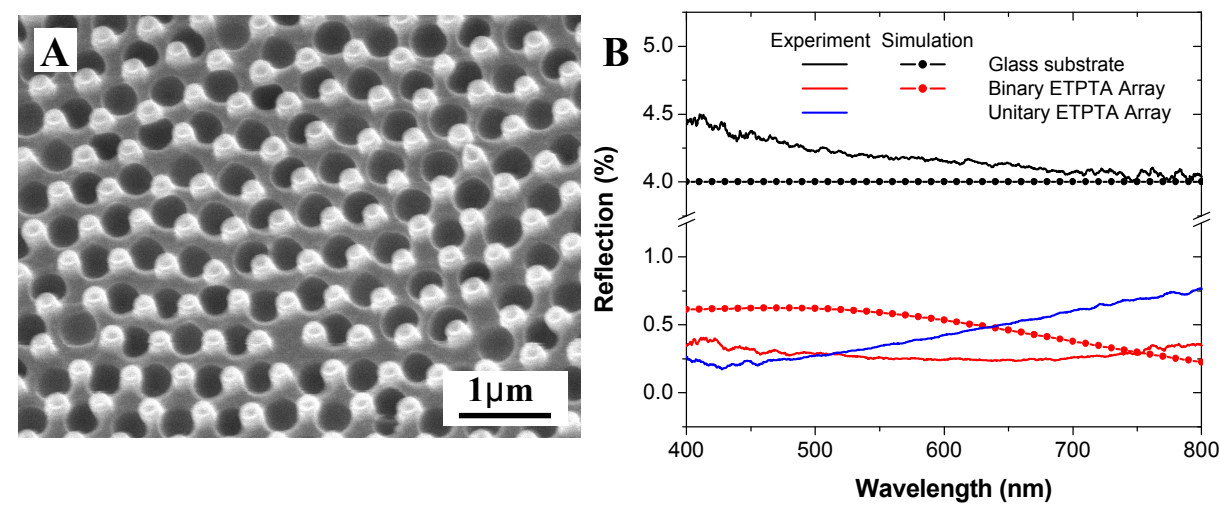

Figure 17. (A) SEM image of a templated polymer binary nipple-dimple ARC. (B) Normal-incidence optical reflection and RCWA simulation from the templated binary ARC, an unitary ARC, and a bare glass substrate.

\section{Fabrication and Characterization of Solar Cells with Optimal Moth-Eye ARCs}

We have successfully doped n-type single-crystalline silicon wafers with templated moth-eye microstructures with p-type dopants (spin-on dopants, Honeywell Electronic Materials). Unfortunately, the electron-beam evaporator at UF, which is needed to metalize the silicon substrates to create electrodes for testing the performance of silicon solar cells, was down since October 2010. Fortunately, we have collaborated with Dr. Jiangeng Xue's group in Material Sciences and Engineering at UF and Emcore Corporation to evaluate the effect of antireflection performance of transparent moth-eye ARCs on the solar cell performance of organic photovoltaics (Dr. Xue) and high-performance III-V PVs (Emcore). Preliminary results show that a templated polymer moth-eye ARC (not optimized yet) can increase the power conversion efficiency of polymer (P3HT:PCBM) bulk-heterojunction solar cell by $\sim 10 \%$ under the standard AM1.5 illumination. The performance of the optimized ARCs on GaAs PVs is still under investigation. 


\section{$\underline{\text { Summary and Conclusions }}$}

An inexpensive, effective anti-reflective coating has been developed at the University of Florida to significantly enhance the absorption of light by silicon in solar cells. This coating has nano-scale features, and its microstructure mimics that of various night active insects (e.g. "moth eye"). Samples of silicon having this coating were exposed either to various combinations of temperature and humidity or to gamma irradiation $\left({ }^{60} \mathrm{Co}\right)$ at the Savannah River National Laboratory, and to a pulsed broad spectrum UV light and to $532 \mathrm{~nm}$ laser light at the University of Florida. The anti-reflective properties of the coatings were unaffected by any of these environmental stresses, and the microstructure of the coating was also unaffected. In fact, the reflectivity of the gamma irradiated ARC became lower at wavelengths between 400 and $1000 \mathrm{~nm}$. These results show that this coating is robust and should be tested in actual systems exposed to either weather or a space environment. Structural details of the ARCs were studied to optimize their performance. Square arrays perform better than hexagonal arrays- the natural moth-eye coating is indeed a square array. The optimal depth of the templated nanopillars in the ARC was found. A wet etching technology for ARC formation was developed that would be less expensive and much faster than dry etching. Theoretical modeling revealed that dimple arrays should perform better than nipple arrays. A way of fabricating both dimple and nipple arrays having the same length was developed, and in fact the dimple arrays did perform better than the nipple arrays, agreeing with the model. We believe the commercial viability of the technology is feasible, since the technology is scalable and inexpensive. It is also compatible with current industrial fabrication of solar cells. 


\section{$\underline{\text { References }}$}

(1). Basic Research Needs for Solar Energy Utilization. (US Department of Energy Report of the Basic Energy Sciences Workshop on Solar Energy Utilization, 2005).

(2). Luque, A. \& Hegedus, S. Handbook of Photovoltaic Science and Engineering. (John Wiley \& Sons, West Sussex, 2003).

(3). Poortmans, J. \& Arkhipov, V. Thin Films Solar cells: Fabrication, Characterization and Applications. (John Wiley \& Sons, Chichester, 2006).

(4). Doshi, P., Jellison, G. E. \& Rohatgi, A. Characterization and optimization of absorbing plasma-enhanced chemical vapor deposited antireflection coatings for silicon photovoltaics. Appl. Opt. 36, 7826-7837 (1997).

(5). Gombert, A. et al. Subwavelength-structured antireflective surfaces on glass. Thin Solid Films 351, 73-78 (1999).

(6). Prevo, B. G., Hon, E. W. \& Velev, O. D. Assembly and characterization of colloid-based antireflective coatings on multicrystalline silicon solar cells. J. Mater. Chem. 17, 791-799 (2007).

(7). Oliva, E., Dimroth, F. \& Bett, A. W. GaAs converters for high power densities of laser illumination. Prog. Photovolt.: Res. Appl. 16, 289-295 (2008).

(8). Luca, S. et al. GaSb crystals and wafers-for photovoltaic devices. J. Solar Energy Eng. 129, 304-313 (2007).

(9). Walheim, S., Schaffer, E., Mlynek, J. \& Steiner, U. Nanophase-separated polymer films as high-performance antireflection coatings. Science 283, 520-522 (1999).

(10). Hiller, J., Mendelsohn, J. D. \& Rubner, M. F. Reversibly erasable nanoporous antireflection coatings from polyelectrolyte multilayers. Nat. Mater. 1, 59-63 (2002).

(11). Ibn-Elhaj, M. \& Schadt, M. Optical polymer thin films with isotropic and anisotropic nano-corrugated surface topologies. Nature 410, 796-799 (2001).

(12). Schulz, U. Review of modern techniques to generate antireflective properties on thermoplastic polymers. Appl. Opt. 45, 1608-1618 (2006).

(13). Jiang, H., Yu, K. \& Wang, Y. C. Antireflective structures via spin casting of polymer latex. Opt. Lett. 32, 575-577 (2007).

(14). Xi, J. Q. et al. Optical thin-film materials with low refractive index for broadband elimination of Fresnel reflection. Nat. Photonics 1, 176-179 (2007).

(15). Zhao, Y., Wang, J. S. \& Mao, G. Z. Colloidal subwavelength nanostructures for antireflection optical coatings. Opt. Lett. 30, 1885-1887 (2005).

(16). Lee, D., Rubner, M. F. \& Cohen, R. E. All-nanoparticle thin-film coatings. Nano Lett. 6, 2305-2312 (2006).

(17). Zhang, X. T. et al. Self-cleaning particle coating with antireflection properties. Chem. Mater. 17, 696-700 (2005).

(18). Huang, Y. F. et al. Improved broadband and quasi-omnidirectional anti-reflection properties with biomimetic silicon nanostructures. Nat. Nanotech. 2, 770-774 (2007).

(19). Stavenga, D. G., Foletti, S., Palasantzas, G. \& Arikawa, K. Light on the moth-eye corneal nipple array of butterflies. Proc. R. Soc. B 273, 661-667 (2006).

(20). Srinivasarao, M. Nano-optics in the biological world: Beetles, butterflies, birds, and moths. Chem. Rev. 99, 1935-1961 (1999).

(21). Vukusic, P. \& Sambles, J. R. Photonic structures in biology. Nature 424, 852-855 (2003). 
(22). Kanamori, Y., Sasaki, M. \& Hane, K. Broadband antireflection gratings fabricated upon silicon substrates. Opt. Lett. 24, 1422-1424 (1999).

(23). Yu, Z. N., Gao, H., Wu, W., Ge, H. X. \& Chou, S. Y. Fabrication of large area subwavelength antireflection structures on Si using trilayer resist nanoimprint lithography and liftoff. J. Vac. Sci. Technol. B 21, 2874-2877 (2003).

(24). Clapham, P. B. \& Hutley, M. C. Reduction of Lens Reflection by Moth Eye Principle. Nature 244, 281-282 (1973).

(25). Hadobas, K., Kirsch, S., Carl, A., Acet, M. \& Wassermann, E. F. Reflection properties of nanostructure-arrayed silicon surfaces. Nanotechnology 11, 161-164 (2000).

(26). Lalanne, P. \& Morris, G. M. Antireflection behavior of silicon subwavelength periodic structures for visible light. Nanotechnology 8, 53-56 (1997).

(27). Sai, H. et al. Antireflective subwavelength structures on crystalline Si fabricated using directly formed anodic porous alumina masks. Appl. Phys. Lett. 88, 201116 (2006).

(28). Wang, S., Yu, X. Z. \& Fan, H. T. Simple lithographic approach for subwavelength structure antireflection. Appl. Phys. Lett. 91, 061105 (2007).

(29). Jiang, P. \& McFarland, M. J. Large-scale fabrication of wafer-size colloidal crystals, macroporous polymers and nanocomposites by spin-coating. J. Am. Chem. Soc. 126, 13778-13786 (2004).

(30). Min, W. L., Betancourt, A. P., Jiang, P. \& Jiang, B. Bioinspired broadband antireflection coatings on GaSb. Appl. Phys. Lett. 92, 141109 (2008).

(31). Min, W. L., Jiang, B. \& Jiang, P. Bioinspired Self-Cleaning Antireflection Coatings. Adv. Mater. 20, 3914-3918 (2008).

(32). Min, W. L., Jiang, P. \& Jiang, B. Large-scale assembly of colloidal nanoparticles and fabrication of periodic subwavelength structures. Nanotechnology 19, 475604 (2008).

(33). Sun, C. H., Gonzalez, A., Linn, N. C., Jiang, P. \& Jiang, B. Templated biomimetic multifunctional coatings. Appl. Phys. Lett. 92, 051107 (2008).

(34). Sun, C. H., Ho, B. J., Jiang, B. \& Jiang, P. Biomimetic subwavelength antireflective gratings on GaAs. Opt. Lett. 33, 2224-2226 (2008).

(35). Sun, C. H., Jiang, P. \& Jiang, B. Broadband moth-eye antireflection coatings on silicon. Appl. Phys. Lett. 92, 061112 (2008).

(36). Sun, C. H., Linn, N. C., Jiang, B. \& Jiang, P. Minireview on self-assembled broadband antireflection coatings on crystalline silicon. European J. Inorg. Chem. (2007).

(37). Sun, C. H., Linn, N. C., Min, W. L., Jiang, B. \& Jiang, P. Large-scale assembly of periodic nanostructures with metastable square lattices. J. Vac. Sci. Technol. B 27, 10431047 (2009).

(38). Sun, C. H., Min, W. L., Linn, N. C., Jiang, P. \& Jiang, B. Templated fabrication of large area subwavelength antireflection gratings on silicon. Appl. Phys. Lett. 91, 231105 (2007).

(39). Linn, N. C., Sun, C. H., Jiang, P. \& Jiang, B. Self-assembled biomimetic antireflection coatings. Appl. Phys. Lett. 91, 101108 (2007).

(40). Jiang, P. Wafer-scale fabrication of periodic polymer attolitre microvial arrays. Chem. Commun., 1699-1701 (2005).

(41). Jiang, P. Large-scale fabrication of periodic nanostructured materials by using hexagonal non-close-packed colloidal crystals as templates. Langmuir 22, 3955-3958 (2006).

(42). Jiang, P. Surface-templated nanostructured films with two-dimensional ordered arrays of voids. Angew. Chem. Int. Ed. 43, 5625-5628 (2004). 
(43). Jiang, P. \& McFarland, M. J. Wafer-scale periodic nanohole arrays templated from twodimensional nonclose-packed colloidal crystals. J. Am. Chem. Soc. 127, 3710-3711 (2005).

(44). Jiang, P., Prasad, T., McFarland, M. J. \& Colvin, V. L. Two-dimensional nonclosepacked colloidal crystals formed by spincoating. Appl. Phys. Lett. 89, 011908 (2006).

(45). Stober, W., Fink, A. \& Bohn, E. Controlled Growth of Monodisperse Silica Spheres in Micron Size Range. J. Colloid Interf. Sci. 26, 62-\& (1968).

(46). Pieranski, P. Colloidal crystals. Contemp. Phys. 24, 25-73 (1983).

(47). Jiang, P., Bertone, J. F., Hwang, K. S. \& Colvin, V. L. Single-crystal colloidal multilayers of controlled thickness. Chem. Mater. 11, 2132-2140 (1999). 